Editorials

\title{
Mechanisms and Management of Chronic Pain
}

\author{
Victor V. Chaban \\ Charles R. Drew University of Medicine and Science, University of California Los Angeles, USA
}

\author{
Article history \\ Received: 23-12-2020 \\ Revised: 02-01-2021 \\ Accepted: 14-01-2021 \\ Email: victorchaban@cdrewu.edu
}

\begin{abstract}
Chronic pain symptoms account up to half of all health care visits, afflicts $>10 \%$ of US adults, at a higher prevalence in women with current analgesic drugs rarely provide enough efficacy in the absence of serious side effects. Chronic pain is also the root cause of the national opioid health crisis, which adds to health care costs and deaths. Thus, new pain therapies based on detailed understanding of nociceptive mechanisms are needed as alternatives to opioid analgesics and are of great societal importance. Chronic pain is one of the most prevalent human health problems that often is associated by the concomitant decline in cognitive and motor functions. Pain is strongly associated with other diseases that can lack of awareness to its pathology. Despite a successful reduction of pain with available medications, majority of treated patients were seeking professional help again. The average time duration between the onset of pain symptoms and the diagnosis is couple of years despite the fact that majority of patients with chronic pain suffer every day. Efficacious and reliable therapeutic intervention is still unavailable despite the tremendous economic burden imposed on healthcare to treat many diseases associated with chronic pain.
\end{abstract}

Keywords: Chronic Pain, Therapeutics, Research
The complex interplay between diverse group of nociceptive mediators, genetic imprint and environmental factors may ultimately determine the outcome of clinical etiology and progression of therapy. Current pharmacologic management of chronic pain has been notoriously difficult and unsatisfactory. Trials with conventional analgesics (e.g., NSAIDs, acetaminophen, aspirin, opioids) did not significantly improve patient's symptoms. However, patients with comorbid anxiety and/or depression due to chronic nociception may benefit from anxiolytic (benzodiazepine) or antidepressant drugs (TCAs, SSRI and SNRI). Some drugs used to manage neuropathic pain (gabapentin, pregabalin) still require confirmation of their efficacy in managing visceral pain. Despite the recent moderate successes of eluxadoline and linaclotide, most drugs are either ineffective in relieving visceral pain or cause excessive GI-related side effects (e.g., lubiprostone, loperamide and the 5-HT3 antagonists alosetron, granisetron and ondansetron). It is well- accepted that visceral sensitivity can be modulated by neurotransmitters, such as Acetylcholine (ACh), Adenosine Triphosphate (ATP) and 5Hydroxytryptamine (5-HT, serotonin) released by lining of different visceral organs. Therefore, chronic hypersensitivity may result from physiological changes of primary afferents intercellular communication through these mediators and can be modulated by new generation therapeutics targeting them.

Spinal interneurons integrate noxious and innocuous sensory inputs from dorsal root ganglia to the dorsal horn and gate their access to spinal projection neurons, as first postulated by the Gate Control Theory of pain processing (Melzack and Wall, 1965). Although specific mechanisms within dorsal horn circuits have been identified and characterized, the diversity of interneuron subtypes, together with limited knowledge on their neurochemistry, precludes comprehensive neuronal network mapping. To change the paradigm in chronic pain treatment it is important to generate critical new data on the functional connectivity of dorsal root to spinal horn projections and their neuroplasticity under visceral chronic pain conditions.

Acute pain often progresses to chronic. In addition, alterations in the limbic and sympathetic nervous systems and hypothalamic-pituitary-adrenal axis mediate a cycle of hypervigilance for pain sensations from different organs, which can lead to descending induction of pathologic changes in these organs (Chaban, 2016; 2020). Because of the inherent subjectivity to pain, there is a wide disparity among patients in their experience. 
There is also a tension between the subjectivity of the patient's pain tolerance and the findings of the clinician that are proportionate to the patient's complaints. Therefore, an important focus of pain management must include the deep assessment of a patient's health history and quality of life that include their emotional, physical and cognitive states. Chronic pain management is a major public health care challenge. Sensitivity to pain remains long after tissue healing. The discovery of the neurochemical mechanisms that maintain chronic pain hypersensitivity is needed for a better treatment. A multicomponent conceptual model of chronic visceral pain must involve physiologic, cognitive and behavior factors necessary for developing new effective therapies for previously unrecognized chronic pain mechanisms and reveal peripheral and central targets for potential non-opioid therapies, contributing to development of strategies to combat the 'opioid epidemic' caused by overuse/misuse of opioid analgesics.

\section{References}

Chaban, V. (2016). Unraveling the Enigma of Visceral Pain. New York: Nova Publishers, ISBN: 97863489-430.

Chaban, V. (2020). Calcium Signaling and Nervous System. Overview and Directions for Research. Nova Medicine and Health, New York, ISBN: 9781-53618-403.

Melzack, R., \& Wall, P. D. (1965). Pain mechanisms: a new theory. Science, 150(3699), 971-979. 